\section{Cuidar em tempos da COVID-19: lições aprendidas entre a ciência e a sociedade}

\author{
Caring in the age of COVID-19: lessons from \\ science and society
}

\section{Cuidar en tiempos del COVID-19: lecciones aprendidas entre la ciencia y la sociedad}

\author{
Claudia Teresa Vieira de Souza 1 \\ Clarice Silva de Santana 1 \\ Patrícia Ferreira 2 \\ João Arriscado Nunes 2 \\ Maria de Lourdes Benamor Teixeira 1 \\ Maria Isabel Fragoso da Silveira Gouvêa 1
}

\section{Resumo}

Esta comunicação é centrada na nossa pesquisa e intervenção em promoção da saúde com pacientes e comunidades afetadas por diversas doenças infecciosas, no projeto Plataforma de Saberes. Esse projeto é desenvolvido no Instituto Nacional de Infectologia Evandro Chagas, Fundação Oswaldo Cruz, no Rio de Janeiro, Brasil, e promove o envolvimento continuado de pesquisadores e profissionais de saúde na produção colaborativa de conhecimento sobre saúde com pacientes e grupos comunitários, e na sua capacitação como promotores de saúde comunitária. Face ao novo cenário epidêmico, surgiu a demanda de um espaço de diálogo e partilha de conhecimento sobre prevenção da transmissão da COVID-19. Devido à interrupção das atividades regulares do Plataforma de Saberes, foi promovida uma estratégia que servisse de elo integrador entre ciência e sociedade e ajudasse a superar o isolamento social imposto pela pandemia da COVID-19. O grupo de WhatsApp possibilitou aos integrantes do projeto participarem por meio de formulação de perguntas (áudio) sobre a COVID-19, por intermédio da mediação dos líderes de grupos comunitários. Os principais questionamentos dos participantes estão relacionados com fatores de risco; formas de transmissão; imunidade ao novo vírus; cuidados relacionados à prevenção, sintomas e tratamento; COVID- 19 e vacina da influenza. O material educativo Cuidar em tempos da COVID-19 foi produzido e compartilhado com os líderes comunitários e disponibilizado aos respectivos grupos, e veio a ser disseminado nas suas comunidades como resposta a uma demanda de conhecimento que responde às preocupações vividas pelos que já se encontram em situação de vulnerabilidade estrutural.

\author{
Correspondência \\ C. T. V. Souza \\ Fundação Oswaldo Cruz. \\ Av. Brasil 4365, Rio de Janeiro, RJ 21040-900, Brasil. \\ claudiateresa.souza@fiocruz.br \\ 1 Fundação Oswaldo Cruz, Rio de Janeiro, Brasil. \\ 2 Centro de Estudos Sociais, Universidade de Coimbra, Coimbra, \\ Portugal.
}




\section{Introdução}

Em consonância com as diretrizes internacionais, as medidas de prevenção preconizadas pelo Ministério da Saúde do Brasil para minimizar a transmissibilidade da COVID-19 em locais com transmissão comunitária incluem o distanciamento social 1,2.

Face à exposição ao risco de contágio e à incapacidade de aceder a cuidados dirigidos a outros problemas de saúde que podem assim ser agravados, situações de vulnerabilidade estrutural ${ }^{3}$ poderão ser ampliadas no contexto da pandemia da COVID-19. Importa, por isso, atuar sobre as formas de articulação entre o conhecimento científico relacionado com a prevenção da transmissão nas comunidades mais vulneráveis e a continuidade da promoção da sua qualidade de vida. Aspectos relacionados com a literacia da saúde e a dificuldade de compreensão das indicações divulgadas pelas autoridades de saúde, com as condições precárias de saneamento básico e acesso à água canalizada, ou de acesso a cuidados de saúde em comunidades carentes requerem, assim, uma atenção urgente que deve ser integrada na promoção das relações de cuidado individual e comunitário perante os desafios presentes desta epidemia.

O projeto Plataforma de Saberes: Envolvimento e Participação da Comunidade em Práticas Inovadoras de Promoção da Saúde e Produção de Conhecimento é desenvolvido, desde 2012, pelo Laboratório de Pesquisa em Epidemiologia e Determinação Social da Saúde (LAP-EPIDSS) do Instituto Nacional de Infectologia Evandro Chagas, Fundação Oswaldo Cruz (INI/Fiocruz), no Rio de Janeiro, Brasil, com a colaboração do Centro de Estudos Sociais da Universidade de Coimbra. O projeto busca construir novas práticas de promoção da saúde e formas de produção colaborativa de conhecimento entre epidemiologistas, cientistas sociais, infectologistas, outros profissionais de saúde, pacientes e membros de grupos comunitários, desenvolvendo ações em saúde de interesse coletivo, possibilitando uma participação ativa da sociedade, principalmente no controle das doenças infecciosas (infecção por HIV, doença de Chagas, entre outras), crônico-degenerativas (como neoplasias) e outros agravos à saúde e de importância sanitária, que impactam na qualidade de vida da população. As principais atividades envolvem palestras educativas sobre temas de interesse dos participantes, visitas a espaços científicos, oficinas de geração de renda e realização de cursos de extensão orientados para as relações entre a ciência e a sociedade em espaços de ensino, pesquisa e cuidado.

O Plataforma de Saberes tem como parceiros sociais a Associação Lutando para Viver Amigos do INI (ALCA-INI), que presta apoio aos pacientes em tratamento de doenças infecciosas no INI e seus familiares; Clube das Amigas da Mama (CAM), grupo de mulheres que vivenciam e/ou vivenciaram o câncer de mama e que incentiva uma rede de apoio para o enfrentamento desta doença; e o Comitê Comunitário Assessor do Centro de Pesquisa Clínica de HIV/AIDS do Hospital Geral de Nova Iguaçu (CCA-HGNI), que assegura a interface entre os investigadores e a comunidade na prevenção e tratamento do HIV/aids. Esses 3 grupos participam ativamente da divulgação das ações do projeto.

Os participantes do projeto já vivem situações de vulnerabilidade estrutural 3 acentuada pela exclusão social, pelas dificuldades de acesso a cuidados de saúde para vigilância e controle de outros agravos à saúde e bem-estar, pelas lutas diárias contra as desigualdades de saúde que as atividades coletivas promovidas pelo projeto procuram apoiar. A maioria dos participantes vê a sua vulnerabilidade potencializada pela COVID-19, por serem portadores de doenças infecciosas e/ou crônicas.

Os grupos comunitários demandam, no contexto das ações do projeto, formas de acesso ao conhecimento científico relacionadas a situações e processos que podem afetar de maneira severa ou contribuir para o agravamento da sua condição de saúde e das pessoas com quem vivem e convivem. Essa atuação da comunidade promove o envolvimento dos pesquisadores do projeto com as experiências de procura por melhores condições de saúde, com base em formas de relação horizontal e assentes nas preocupações e problemas da comunidade relacionados com a saúde e qualidade de vida. É nesse contexto que quer esses grupos, que os pesquisadores se apropriem informalmente do papel de Promotores da Saúde Comunitária.

A demanda mais recente da comunidade refere-se ao conhecimento sobre a COVID-19, dada a preocupação dos participantes com a circulação de fake news. Para respondermos a essa demanda, organizamos uma roda de conversa intitulada Contribuições dos Promotores da Saúde Comunitária na Prevenção e Controle da COVID-19, prevista para 19 de março de 2020, com a participação de epide- 
miologistas, infectologistas e especialistas em infecção hospitalar, de modo a envolver a comunidade na construção compartilhada de conhecimentos sobre o tema.

A confirmação de transmissão local de SARS-CoV-2, pelas autoridades sanitárias do Rio de Janeiro e a implantação de medidas no quadro da emergência de saúde pública de importância internacional decorrente da COVID-19, incluindo o isolamento social no Estado do Rio de Janeiro, como forma de diminuir a transmissão comunitária 4, tornou o cancelamento do evento inevitável pela preocupação com o distanciamento e o isolamento dos participantes do projeto.

A atenção que dedicamos nesse projeto aos que são vulnerabilizados pela desvalorização das suas experiências e dos seus conhecimentos, levou-nos a criar um espaço de ação e de resposta relacionadas com situações de doença, de vulnerabilidade e necessidades de formas diversas de cuidado com a situação de epidemia ${ }^{5}$. As relações de confiança e de cuidado construídas pelas atividades de promoção da saúde nos fizeram pensar, perante o isolamento social imposto, em utilizar o WhatsApp como ferramenta de comunicação para enfrentar coletivamente os desafios colocados, mantendo vivas as atividades do projeto agora num espaço virtual.

O objetivo desta comunicação é divulgar e apresentar a construção desse espaço de diálogo e troca de experiências em um ambiente virtual com o qual os participantes estavam já familiarizados quer no âmbito do projeto, quer nas suas relações pessoais, na construção de conhecimento em COVID-19.

\section{Metodologia}

Esta comunicação apresenta o relato de experiência de uma ação em saúde por meio da construção coletiva de um material educativo como contribuição na prevenção e enfrentamento da COVID-19.

Procuramos valorizar os questionamentos partilhados buscando responder com o grupo aos novos desafios impostos. Utilizamos o WhatsApp como ferramenta de construção participativa visando a promover o empoderamento individual, a participação coletiva dos envolvidos no projeto e buscando fomentar o engajamento comunitário com base na utilização de metodologias participativas 6,7,8.

O grupo de WhatsApp intitulado Cuidar para Evitar a COVID-19 procurou viabilizar a construção da atividade com a equipe do LAP-EPIDSS e com 3 grupos comunitários (ALCA-INI, CAM, CCA-HGNI) como mediadores entre o projeto e os membros de seus grupos. Optamos por usar esse aplicativo por ser o meio virtual mais utilizado por esses grupos sociais para se comunicarem com os seus respectivos membros.

Consideramos líderes as pessoas que participam e se dedicam aos seus grupos comunitários, inspirando confiança e oferecendo atenção e cuidado individualizado, e buscando soluções com os membros de sua comunidade. Assim, foi apresentada aos 4 líderes de grupo comunitário (LGC) participantes do projeto a proposta de construção coletiva de um material educativo sobre COVID-19, tendo o WhatsApp como cenário para esta construção. Visto que os grupos comunitários representados pelos seus LGC têm um grupo no WhatsApp, a proposta era que partilhassem com os participantes a questão: "Qual informação sobre o novo Coronavírus-19 você acha importante divulgar para a comunidade?”.

A pesquisa ocorreu entre os dias 23 e 26 de março de 2020. Incluímos no grupo do WhatsApp uma mensagem com a questão norteadora da proposta e um áudio para esclarecer a atividade para os LGC participantes do Plataforma de Saberes, como forma de promover o acesso e a compreensão de todos os envolvidos.

O projeto teve a aprovação do Comitê de Ética em Pesquisa do INI/Fiocruz (CAAE no 88434218.6.0000.5262). 


\section{Resultados}

O grupo do WhatsApp Cuidar para Evitar a COVID- 19 foi criado em 23 de março de 2020. Foi composto por 5 membros da equipe do Plataforma de Saberes e 4 LGC de grupos comunitários que participam, ativamente, das atividades do projeto. Cabe mencionar que houve a participação de 2 líderes da ALCA-INI. Todos aceitaram participar e manifestaram que a iniciativa foi uma forma de se sentirem inseridos e valorizados no processo de produção e partilha de conhecimento em COVID-19.

Os principais questionamentos dos participantes foram relacionados aos fatores de risco, formas de transmissão, imunidade ao novo vírus, cuidados relacionados à prevenção, sintomas e tratamento, COVID-19 e vacina da influenza.

Após o levantamento das perguntas apontadas pelos participantes de cada grupo, a equipe elaborou as respostas baseadas em sites oficiais da Organização Mundial da Saúde, do Centro para o Controle e Prevenção de Doenças (Centers for Disease Control and Prevention, Estados Unidos), da Sociedade Brasileira de Infectologia e do Ministério da Saúde.

Pela urgência em oferecer o feedback, a consolidação e o layout do material Cuidar em Tempos da COVID-19 foram compartilhados com os LGC dos grupos comunitários participantes. Após revisão e avaliação pelos LGC, principalmente quanto ao conteúdo, linguagem utilizada e apresentação, o material foi aprovado, sendo disponibilizado pelos líderes aos seus respectivos grupos comunitários, totalizando, aproximadamente, 100 pessoas que tiveram acesso ao material produzido. Alguns LGC divulgaram também o material produzido noutros estados do Brasil (Pernambuco, Bahia, Espírito Santo e São Paulo). Membros da equipe do projeto solicitaram também a divulgação em seus grupos de trabalho (instituições de saúde e ensino), além dos grupos de amigos.

Acreditamos que esse material alcançou um número ampliado de pessoas em relação ao estimado inicialmente, o que tem sido comum na trajetória do projeto. O material produzido foi inserido no Repositório Institucional da Fundação Oswaldo Cruz, o Arca (https://www.arca.fiocruz.br/handle/ icict/41236).

\section{Discussão}

O Plataforma de Saberes tem uma longa trajetória de investigação-ação centrada na produção colaborativa de conhecimento em contexto de pesquisa clínica, epidemiológica e social em doenças infecciosas e de criação de práticas inovadoras na promoção da saúde. É, também, um espaço agregador e construtor de relações de confiança e de cuidado por meio das ações de promoção da saúde.

A distância e o isolamento, apesar de serem medidas necessárias, passaram a nos preocupar porque o isolamento e a exclusão social são parte integrante da vulnerabilidade estrutural 3 que afeta os participantes (e suas comunidades). Baseando-se no diálogo e na troca de experiências em um ambiente virtual, construímos uma estratégia para superar o distanciamento entre os participantes do projeto e apoiar as demandas de conhecimento sobre o enfrentamento da COVID-19.

Essa iniciativa, não prevista inicialmente nas atividades do Plataforma de Saberes, surgiu como resposta à necessidade de intervenção imediata justificada pelas características da COVID-19.

O grupo de WhatsApp Cuidar para Evitar constituiu um espaço de diálogo e partilha de conhecimento na prevenção da COVID-19, mobilizando todos os atores sociais envolvidos neste processo participativo. Os relatos individuais partilhados na rede de solidariedade que o projeto promove permitiram atenuar, como esperávamos, o isolamento social sentido por muitos participantes e continuou a estreitar as relações como forma de cuidar. Cuidar é, no entendimento do Plataforma de Saberes, uma forma de relação e uma postura ética na pesquisa e intervenção, que visibiliza a relevância das atitudes e valores morais das relações afetivas e da nossa responsabilidade face a um envolvimento de compreensão e respeito por quem se encontra vulnerável 9,10.

Esse novo olhar sobre o cuidado problematiza e procura contribuir para a transformação social do papel dos profissionais de saúde 11 , mas também da relação de todos os participantes por meio de novas experiências de cuidar em condições de exceção.

Utilizar o diálogo, a criatividade e a escuta atenta às demandas dos participantes para a promoção dessa forma de cuidado, que é a tradução do que se expressa como saúde no projeto, é algo inerente no 
planejamento das ações propostas. Essa experiência reforça a proposta de que para promover a saúde necessitamos promover mutuamente um cuidado empoderador e o bem-estar. Produzir esse cuidado contribui para a sobrevivência e o florescimento de quem cuida e de quem recebe o cuidado, e para o reconhecimento da interdependência como condição de uma vida digna.

Face à necessidade urgente de novas formas de intervenção na epidemia da COVID-19, acreditamos que essa experiência poderá ampliar o escopo de futuras ações de saúde pública assentes no compromisso científico com as lutas pela saúde dessas comunidades e na força comunitária que decorre desse envolvimento, força essa traduzida na ação solidária dos promotores da saúde comunitária no controle da transmissão da doença nas suas comunidades, mas também de um aprendizado e de um processo de produção de novos saberes e capacidades ampliados na sua experiência e no diálogo com pesquisadores e profissionais 12,13 .

Conclui-se que as dimensões sociais e políticas complexas da pandemia de COVID-19 precisam integrar essa construção ampliada da promoção da saúde e da saúde pública.

Pretendemos dar continuidade a esta experiência como espaço de diálogo, construção de conhecimentos e de cuidado com base na prevenção e promoção da saúde, pois representa o nosso posicionamento ético e o compromisso com o devir dos que vivem esta epidemia.

Esta breve comunicação serve, também, de reflexão para um olhar sobre os novos processos de determinação social da saúde e da produção de novos conhecimentos decorrentes do encontro entre conhecimento científico e conhecimentos nascidos da experiência em contexto de emergência sanitária.

\section{Colaboradores}

C. T. V. Souza, C. S. Santana e P. Ferreira contribuíram na concepção e delineamento do estudo, análise, coleta e interpretação dos dados, redação, revisão crítica relevante do conteúdo intelectual e aprovação da versão final do manuscrito, incluindo a garantia de sua precisão e integridade. J. A. Nunes, M. L. B. Teixeira e M. I. F. S. Gouvêa contribuíram com a análise, coleta e interpretação dos dados, redação, revisão crítica relevante do conteúdo intelectual e aprovação da versão final do manuscrito, incluindo a garantia de sua precisão e integridade.

\section{Informações adicionais}

ORCID: Claudia Teresa Vieira de Souza (00000003-3208-722X); Clarice Silva de Santana (00000002-6378-4020); Patrícia Ferreira (0000-00016229-1091); João Arriscado Nunes (0000-00030109-8268); Maria de Lourdes Benamor Teixeira (0000-0002-6896-0089); Maria Isabel Fragoso da Silveira Gouvêa (0000-0002-1772-1942).

\section{Agradecimentos}

Aos líderes e representantes de grupos comunitários que participaram da pesquisa: Marcos Maurício Braga Cardozo, Valdinei da Silva, Joelma Araújo Tavares Ali, Juciara Nicomedio e demais participantes do projeto Plataforma de Saberes. Aos membros da equipe do Plataforma de Saberes: José Liporage Teixeira (farmacêutico) e Aline Fabrícia Santos da Silva Bistene, Ana Carolina de Freitas Guimarães e Karina Diniz Pinto (bolsistas). 


\section{Referências}

1. Secretaria de Atenção Primária à Saúde, Ministério da Saúde. Protocolo de manejo clínico do coronavírus (COVID-19) na atenção primária à saúde; março de 2020. https://coronavi rus.saude.gov.br/manejo-clinico-e-tratamento (acessado em 29/Abr/2020).

2. World Health Organization. Coronavirus disease (COVID-19) pandemic. https://www. who.int/emergencies/diseases/novel-corona virus-2019 (acessado em 29/Abr/2020).

3. Bourgois P, Holmes SM, Sue K, Quesada J. Strutural vulnerability: operationalizing the concept to adress health disparities in clinical care. Acad Med 2017; 3:299-307.

4. Rio de Janeiro. Decreto no 46.973, de 16 de março de 2020. Reconhece a situação de emergência na saúde pública do Estado do Rio de Janeiro em razão do contágio e adota medidas de enfrentamento da propagação decorrente do novo coronavírus (COVID-19); e dá outras providências. Diário Oficial do Estado do Rio de Janeiro 2020; 17 mar.

5. Ferreira P, Souza CTV, Santana CS, Nunes JA. Caring in times of COVID-19. Somatosphere: Science, Medicine, and Anthropology 2020; http://somatosphere.net/2020/caringin-times-of-covid-19.html/ (acessado em 17/ Abr/2020).

6. Lima ICV, Galvão MTG, Pedrosa SC, Cunha $\mathrm{GH}$, Costa AKB. Uso do aplicativo Whatsapp no acompanhamento em saúde de pessoas com HIV: uma análise temática. Esc Anna Nery Rev Enferm 2018; 22:e20170429.
7. Silva RC. Metodologias participativas para trabalhos de promoção de saúde e cidadania. São Paulo: Vetor; 2002.

8. Queiroz AG, Couto ACP. Metodologia participativa, subjetividade individual e social: facilitação de reuniões de moradores em Residências Terapêuticas. Pesqui Prát Psicossociais $2015 ; 10: 171-8$

9. Waldow VR. Cuidar: expressão humanizadora da enfermagem. 6a Ed. Petrópolis: Editora Vozes; 2012.

10. Waldow VR. Enfermagem: a prática do cuidado sob o ponto de vista filosófico. Investigación en Enfermería: Imagen y Desarrollo 2015; 17:13-25.

11. Barros S, Oliveira MAF, Silva ALA. Práticas inovadoras para o cuidado em saúde. Rev Esc Enferm USP 2007; 41(Esp):815-9.

12. Lima FA, Galimbertti PA. Sentidos da participação social na saúde para lideranças comunitárias e profissionais da Estratégia Saúde da Família do território de Vila União, em Sobral - CE. Physis (Rio J.) 2016; 26:157-75.

13. Santos BS. O fim do império cognitivo. Belo Horizonte: Autêntica Editora; 2019. 


\section{Abstract}

This paper focuses on our research and intervention in health promotion with patients and communities affected by various infectious diseases, in the project Knowledge-Sharing Platform. This project is developed at the National Institute of Infectious Diseases Evandro Chagas in the Oswaldo Cruz Foundation, Rio de Janeiro, Brazil, and promotes the continuing involvement of health researchers and professionals in the collaborative production of knowledge on health with patients and community groups and in their training as community health promoters. The new epidemic scenario created the demand for a space of dialogue and knowledge-sharing on the prevention of COVID-19 transmission. Due to the interruption of regular activities in the Knowledge-Sharing Platform, a strategy was launched to serve as a link between science and society and help overcome the social isolation imposed by the COVID-19 pandemic. The WhatsApp group allowed project members to participate by addressing questions (audio) on COVID-19 through mediation by community group leaders. The main questions by participants related to risk factors, transmission, immunity to the novel coronavirus, care related to prevention, symptoms, and treatment; and COVID-19 and influenza vaccine. The educational material Caring in the Age of COVID-19 was produced and shared with community leaders and distributed to the respective groups and was eventually shared in their communities as a response to a demand for knowledge that responds to the concerns of people already exposed to structural vulnerability.

Citizen Participation in Science and Technology; Health Promotion; Social Isolation; COVID-19

\section{Resumen}

Esta comunicación se centra en nuestra investigación e intervención en la promoción de la salud con pacientes y comunidades afectadas por diversas enfermedades infecciosas, en el proyecto Plataforma de Saberes. Este proyecto está desarrollado en el Instituto Nacional de Infectología Evandro Chagas/Fundación Oswaldo Cruz, en Río de Janeiro, Brasil, y promueve la implicación continuada de investigadores y profesionales de salud en la producción colaborativa de conocimiento sobre salud con pacientes y grupos comunitarios, $y$ en su capacitación como promotores de salud comunitaria. Frente al nuevo escenario epidémico, surgió la demanda de un espacio de diálogo e intercambio de conocimiento sobre la prevención de la transmisión de la COVID-19. Debido a la interrupción de las actividades regulares de la Plataforma de Saberes, se promovió una estrategia que sirviese de vínculo integrador entre ciencia y sociedad y ayudase a superar el aislamiento social impuesto por la pandemia de la COVID-19. El grupo de WhatsApp posibilitó a los integrantes del proyecto, que participaran mediante la formulación de preguntas (audio) sobre la COVID-19, a través de la mediación de los líderes de grupos comunitarios. Los principales cuestionamientos de los participantes se relacionaron con factores de riesgo; formas de transmisión; inmunidad ante el nuevo virus; cuidados relacionados con la prevención, sintomas y tratamiento; COVID-19 y vacuna de la gripe. El material educativo Cuidar em tempos da COVID-19 se produjo y se compartió con los líderes comunitarios y fue puesto a disposición de los respectivos grupos, así como difundido en sus comunidades, en respuesta a una demanda de conocimiento que responde a las preocupaciones vividas por los que ya se encuentran en situación de vulnerabilidad estructural.

Participación Ciudadana en Ciencia y

Tecnología; Promoción de la Salud;

Aislamiento Social; COVID-19
Recebido em 07/Mai/2020

Versão final reapresentada em 17/Mai/2020

Aprovado em 26/Mai/2020 